\title{
L'HOMME L'Homme
}

Revue française d'anthropologie

$170 \mid 2004$

Espèces d'objets

\section{L'objet invisible}

ou le gambit du capitaine

\section{Gaetano Ciarcia}

\section{(2) OpenEdition \\ Journals}

Édition électronique

URL : http://journals.openedition.org/lhomme/24819

DOI : 10.4000/lhomme.24819

ISSN : 1953-8103

Éditeur

Éditions de l'EHESS

\section{Édition imprimée}

Date de publication : 1 juin 2004

Pagination : 199-229

ISSN : 0439-4216

Référence électronique

Gaetano Ciarcia, «L'objet invisible », L'Homme [En ligne], 170 | 2004, mis en ligne le 01 janvier 2006, consulté le 30 avril 2019. URL : http://journals.openedition.org//homme/24819; DOI : 10.4000/ Ihomme.24819 


\title{
L'objet invisible ou le gambit du capitaine
}

\section{Gaetano Ciarcia}

\begin{abstract}
Ainsi l'esclave aveugle obéit à son aveugle maître et, dans son obéissance, le tua. Ainsi le créateur fut occis par la créature. Ainsi la cloche fut trop lourde pour la tour. Ainsi le défaut de la cloche apparut là où le sang l'avait viciée. Ainsi l'orgueil précéda la chute.
\end{abstract}

Herman Melville, Le Campanile

\section{C}

ECI EST L'HISTOIRE du capitaine Amasa Delano qui ne s'aperçoit pas que sur le San Dominick - un bateau négrier espagnol en détresse à qui il porte secours - les Blancs sont devenus les otages des Noirs ${ }^{1}$. Il se rend bien compte que règne sur le navire une inquiétante étrangeté, mais le code pour l'interpréter lui manque. L'intrus, se voulant être le sauveur, est en réalité la dupe que Benito Cereno, le capitaine du San Dominick, luimême tenu en otage, tente désespérément de protéger. Delano essaie pourtant de maîtriser les signes à la fois familiers et incongrus à travers des hypothèses domestiques - par moments il imagine Benito Cereno en ennemi, chef d'un équipage de pirates - qui se révèlent être autant des illusions nécessaires à sa survie que des convictions funestes. Delano est, en effet, confronté à un réel qui lui échappe et qui, pour lui, demeure inconcevable; mais il fait comme s'il ne l'était pas, justement pour se donner l'impression d'évoluer dans un monde sur lequel il pense avoir encore prise. Inconsciemment, il fuit une vérité, celle d'une mutinerie, se cachant derrière les linéaments d'une raison qui ne peut que vaciller face aux épreuves de l'expérience. À travers l'alternance de ses états d'âme, entre confiance et peur, c'est aussi et surtout le refoulé de l'esclavage qui lui cache la révolte en acte de ceux qui devraient occuper la place de prisonniers soumis mais qui apparaissent comme des partenaires à part entière des matelots espagnols. Leur comédie semble bien orchestrée quoique grossière; néanmoins, elle est sur le point d'atteindre la perfection grâce à la participation et la collaboration d'un observateur étranger tout à sa pen-

1. Herman Melville, Benito Cereno, Paris, Flammarion, 1991 [1855] (trad. de Jean-Pierre Naugrette). Je développe ici un passage de mon article "Le goût de la croyance. La dénégation nécessaire et son objet fétiche ", L'Homme, 2003, $166: 175$ sq. 
sée coloniale, qui ne peut donc imaginer l'inversion, encore moins la subversion des signes de la présence à soi.

Benito Cereno c'est aussi l'histoire des déplacements des pièces d'un puzzle toujours en train de se défaire lorsque celui qui s'est pris au jeu tente vainement de l'assembler, essayant de lui donner ou plutôt de lui communiquer une cohérence ${ }^{2}$. Ces pièces-signes qu'Herman Melville dissémine devant les yeux de ses lecteurs et du joueur en question : Amasa Delano, commandant du Bachelor's Delight ${ }^{3}$, navire phoquier américain faisant relâche près de l'îlot désertique de Santa-Maria, au large de l'extrémité méridionale du littoral chilien. Un vaisseau n'arborant aucun pavillon apparait à travers sa lorgnette. Il s'agit du San Dominick, bâtiment espagnol à l'allure surannée, qui à l'époque des faits, en 1799, capte l'attention de l'observateur à cause de :

"... la principale relique de cette grandeur passée [...] cette pièce de poupe en forme d'écusson, dont l'ovale majestueux était gravé aux armes de la Castille et du Léon savamment entrelacées, entourées de médaillons représentant des motifs mythologiques et symboliques, avec, en haut et au centre, un satyre noir, masqué, le pied posé sur le cou prostré d'une figure contorsionnée, également masquée »4.

Dès le début du récit, l'énigme est posée au lecteur par ces figures du satyre noir et de son opprimé, mais le mystère qu'elles représentent est noyé parmi les autres indices montrant clairement un bateau et son équipage, rescapés d'un drame de la mer. Delano se rend à bord du San Dominick:

"Grimpant par la coupée, le visiteur fut aussitôt assiégé par une foule vociférante de Blancs et de Noirs, ces derniers l'emportant toutefois en nombre sur les premiers dans une proportion inattendue, si l'on se souvenait que le navire inconnu entré dans la rade était un négrier. Pourtant, c'est dans une seule langue et comme qui dirait d'une seule voix que l'ensemble débita un même récit de souffrances [...] Le scorbut, allié à la fièvre, avait décimé une bonne partie de l'équipage, emportant plus particulièrement les Espagnols. En doublant le cap Horn ils avaient échappé de justesse au naufrage [...] ; leurs provisions étaient au plus bas ; leur eau pratiquement inexistante... " "5

2. Situation inverse, en quelque sorte, au procédé adopté par Percival Bartlebooth, un des personnages de La Vie, mode d'emploi, de Georges Perec, consistant à effacer les paysages (des marines) qu'il avait peints : après la destruction des tableaux originaux, leurs reconstitutions sous forme de puzzles étaient destinées à être " rapatriées " dans les lieux qui avaient inspiré l'exécution originaire. 3. Le Bachelor's Delight est un des bateaux du boucanier William Ambrose Cowley, dont il est question dans la nouvelle de Melville Les Îles enchantées (1854). Mais ce nom représente aussi la synthèse de deux autres bâtiments traversant l'œuvre melvillienne : le Bachelor (la septième des neuf baleinières croisées par le Pequod sur les traces de Moby Dick; au moment de la rencontre avec le navire d'Achab, à bord du Bachelor - chargé de tonneaux contenant du spermaceti -, les matelots dansent avec des filles polynésiennes. À l'esprit convivial de son capitaine, l'invitant à se désister de son entreprise et à se joindre à la "fête ", Achab oppose un refus dédaigneux) et le Delight (la neuvième baleinière qui, en contraste avec son nom, ayant perdu cinq hommes et un de ses canots, montre prophétiquement aux hommes du Pequod les signes néfastes de son affrontement avec Moby Dick).

4. Hermann Melville, Benito Cereno, op. cit. : 65.

5. Ibid.: 66. 
Le constat surprenant d'un navire utilisé pour la traite où les Blancs et les Noirs associent leurs voix en une seule langue ordonne la découverte d'un ensemble d'êtres humains formant une entité malheureuse mais étrangement solidaire. Les éléments du puzzle acquièrent leurs contours à l'instant même où l'image préalable de l'objet, celle d'un bateau négrier, se dissout ${ }^{6}$. D'ailleurs, c'est la narration des faits qui domine le regard de l'observateur :

«... six autres Noirs; chacun muni d'une hachette rouillée qu'il était occupé à fourbir $[\ldots]$; tandis qu'entre chaque couple gisait une petite pile de hachettes, dont les tranchants rouillés, tournés vers le dehors attendaient la suite de l'opération... $»^{7}$.

Delano voit bien la menace que représentent ces lames prêtes au combat entre les mains libres d'esclaves, mais après avoir été confusément mis au courant de l'histoire du San Dominick, il évolue désormais dans un pays étranger dont il est obligé de comprendre les mœurs. Après le départ du Rover, le canot qui l'a amené à bord du San Dominick, il se retrouve seul. Et la rencontre avec son homologue, censé pouvoir l'informer davantage, ne le sort pas de l'isolement : "En proie à un abattement permanent... ", $^{8}$, l'autre capitaine, Benito Cereno, apparait comme un homme malade, épuisé, ne témoignant d'aucune empathie avec son hôte qui est pourtant en train de porter secours à son bâtiment. Décidément, Benito Cereno n'est pas l'interlocuteur souhaité par Delano ; la personne semble vidée de sa fonction, et son rôle rempli par les gestes obséquieux d'un esclave, Babo, qui, en quelque sorte, lui colle à la peau : «... un Noir de petite stature, au visage primitif, sur lequel, lorsqu'il le levait, comme un chien de berger, vers celui de l'Espagnol, se mêlaient également le chagrin et l'affection "'.

À bord du San Dominick, Delano devient un spectateur, stupéfié et indigné de voir l'autorité des Blancs conspuée par les agressions qu’occasionnellement les matelots espagnols subissent de la part des Noirs. Mais Babo, toujours aux côtés de son capitaine - sans pour autant pouvoir " extirper le demi-lunatique Don Benito de sa nuageuse langueur $»^{10}-$, avec sa conduite de serviteur fidèle et attentionné, contrebalance des comportements qui demeurent ambigus sinon scandaleux aux yeux de l'Américain ${ }^{11}$. Au demeurant, quoique la discipline soit bafouée par des actes très graves, son rétablissement toujours approximatif est perçu par Delano comme le

6. Ici, par contre, la description melvillienne semble faire pièce avec l'entreprise menée par le personnage de Perec (voir supra note 2).

7. Ibid. : 67-68. C'est moi qui souligne.

8. Ibid. : 69-70.

9. Ibid. : 68 .

10. Ibid. : 71 .

11. Jugement renforcé par la présence de figures comme celle d'Atufal, un géant présenté à Delano comme étant un ancien roi africain, qui, enchaîné, doit comparaître toutes les deux heures devant Benito Cereno pour s'excuser d'un acte d'insoumission. Et si le colosse se refuse à demander pardon, il se montre néanmoins obéissant et respectueux devant son " maître ". 
résultat des hésitations incessantes de Benito Cereno. Imprégné de bonnes intentions, le "visiteur " n'a qu'un seul repère, un seul guide: Babo, le bras droit et la voix de son maître, qui incarne et interprète la hiérarchie du monde; il est rassurant, à sa place - peut-être un peu trop.

\section{Des signes noués}

Dans la tentative d'arracher à la dérive cette communauté minée par la mésaventure, Delano traverse le navire en détresse, à la recherche d'un monde et d'un ordre perdus. Crainte et confiance s'opposent dans son esprit, et si son corps lui transmet une sensation de peur, sa volonté s'empresse de lui restituer une force vacillante:

"Aussi fut-ce à pas comptés que le brave capitaine Delano passa au milieu d'eux, et au moment de leur tourner le dos, tel un homme qui passe par les baguettes, il sentit ses mollets se contracter d'appréhension. Mais lorsqu'il fit volte-face et vit tous les hommes alignés, comme autant de joueurs d'orgue de Barbarie, toujours absorbés par leur besogne et indifférents au monde environnant, il ne put réprimer un sourire face à son récent accès de panique. $»^{12}$

Néanmoins, cet effort de maitrise de soi, aux prises avec une réalité où la persistance de l'impression du danger est constamment démentie par l'incongruité des scènes dont il est le témoin perplexe, n'enlève pas ses doutes à l'observateur ; au contraire il ne fait que les alimenter. Delano multiplie alors les questions, mais celles-ci se heurtent à des réponses évasives, des postures opaques, des attitudes difficilement déchiffrables, parfois inquiétantes, de Benito Cereno, réfractaire, semble-t-il, à toute véritable clarification :

"Ces singulières alternances de courtoisie et de goujaterie chez le capitaine espagnol étaient inexplicables, à moins de retenir l'une des deux hypothèses suivantes - soit la folie douce, soit l'imposture caractérisée. $»^{13}$

«Du même coup, le même comportement qui, en l'occurrence, avait déclenché l'alarme, servait à l'apaiser. En un mot, à peine un soupçon ou une appréhension, aussi fondé qu'il ait pu paraitre alors, qui ne fût désormais, avec autant de fondement apparent, dissipé. $\aleph^{14}$

Le capitaine Delano perçoit les signes (il les voit en tant que symptômes d'un obscur malaise) mais il ne se voit pas, ou il ne veut pas se voir en relation avec les signifiés intrinsèques à leur réitération, et qu'il en vient à confondre avec l'alternance de ses états humoraux. Son observation des faits est en conflit avec son implication dans leur enchaînement. De son côté, Benito Cereno est le Blanc devenu étranger à son semblable en race

12. Ibid. : 81 .

13. Ibid. : 88 .

14. Ibid. : 96.

\section{Gaetano Ciarcia}


comme en grade; il est dépossédé de sa situation pour en devenir l'énigme et le paradoxe :

"Il y avait une différence entre l'idée d'un Don Benito en train de comploter dans les ténèbres sur le sort du capitaine Delano, et celle d'un capitaine Delano insouciant en train d'arranger celui de Don Benito. ${ }^{15}$

À la surface de cette différence, Benito Cereno devient l'objet fantôme, Delano son chercheur désemparé. Alors, faisant preuve d'une mauvaise foi teintée de naïveté, celui qui s'évertue à comprendre utilise la seule position lui paraissant viable pour assurer sa quête confrontée aux supports d'une vérité constamment dérobée :

«... le capitaine Delano, adoptant un air désinvolte et bon enfant, poursuit sa progression non sans lancer de temps à autre un mot plaisant aux nègres et surveiller particulièrement du regard les visages des Blancs çà et là disséminés au milieu des Noirs tels des pions blancs dangereusement égarés sur l'échiquier parmi les rangs de pions adverses $»^{16}$.

Sur les cases de l'échiquier, le capitaine américain a perdu progressivement ses repères et les raisons de son initiative de sauvetage :

«... il lui semblait être dans une contrée isolée à l'intérieur des terres, prisonnier dans quelque château abandonné, avec pour seule occupation de contempler des jardins déserts et de scruter des chemins vagues que ni voiture ni voyageur ne venaient fréquenter $»^{17}$.

L'attente d'un dénouement alimente, presque malgré soi, un regard qui arrive à cerner avec ironie la scène sinistre des événements, comme lorsque Delano surprend Babo en train de raser Benito Cereno à l'aide d'une bien étrange serviette :

«Le château et le lion! s'écria le capitaine Delano - mais, Don Benito, c'est le pavillon espagnol dont vous vous servez là! Il vaut mieux que ce soit moi seulement, et non le roi, qui soit témoin de cela, ajouta-t-il avec un sourire, mais - et il se tourna vers le Noir - c'est tout un, j'imagine, pourvu que les couleurs soient gaies; remarque plaisante qui ne manqua point de titiller le nègre. ${ }^{18}$

Babo, le nègre titillé, est le régisseur de cette contrée dont Delano avec "son air désinvolte et bon enfant " commence à interpréter (au sens théâtral du terme) le mystère ${ }^{19}$. L'épisode où il parle de «son impuissance à sai-

15. Ibid. : 97.

16. Ibid. : $99-100$.

17. Ibid. : 104 .

18. Ibid. : 121

19. Mise en scène qui peut profiter aussi d'une participation spontanée, scellant l'authenticité apparente des comportements; un exemple est donné par l'accueil que, lors de la distribution des vivres et de l'eau exécutée par Delano lui-même, les Noirs réservent à l'échange entre les deux capitaines: "C'est à lui [Benito Cereno], au premier chef, que le capitaine Delano présenta un broc copieusement rempli de ce liquide ; pourtant, malgré sa soif pressante, l'Espagnol n'en avala point .../... 
sir le sens d'un tel nœud $"{ }^{20}$ semble manifester l'intention de participer, en étranger ignare accroché obstinément à la lettre des phénomènes et des objets, la trame qui lui est encore secrète :

" "Qu'êtes-vous en train de nouer là, mon brave?

- Le nœud, répondit l'autre brièvement, sans lever la tête.

- Soit ; mais dans quel but?

- Pour qu'un autre le débrouille", murmura le vieillard, en guise de réponse, faisant courir ses doigts de plus belle, le nœud étant maintenant presque achevé.

Pendant que le capitaine Delano se tenait là à l'observer, tout à coup le vieillard lui lança le nœud en lui disant dans un anglais haché - le premier qu'il entendît sur ce navire - quelque chose comme :

"Débrouillez, tranchez, vite."

Cela fut dit tout bas, mais d'une manière si prompte et ramassée que les longs et lents échanges en espagnol qui avaient précédé et qui suivirent servirent pour ainsi dire à couvrir le bref intermède en anglais [...].

Un nègre d'un certain âge [...] s'avança alors vers le capitaine Delano avec qui comme dirait une mine de notaire. Dans un espagnol convenable, et après un clin d'œil entendu et bonhomme, il l'informa que le vieux faiseur de nœuds était simplet, mais inoffensif, et qu'il jouait souvent ses vieux tours. Le nègre conclut en réclamant le nœud, car bien sûr l'étranger n'en avait que faire. On le lui donna sans autre forme de procès. Le nègre le reçut avec une sorte de courbette, et, tournant le dos, se mit à fouiner dans l'entrelacs tel un agent des douanes cherchant des dentelles. Peu après, avec un terme africain - l'équivalent de «Peuh!» -, il lança le nœud par-dessus bord. " ${ }^{21}$

Le symbole du noud rappelle le thème abordé dans le chapitre XLVII de Moby Dick, "Le tresseur de nattes" où l'allusion allégorique à l'ourdi (à l'instar de la peau tatouée de Queequeg ?) évoquait l'idée d'une quête fatale reliant sans trêve la liberté de l'individu aux amarres de sa condi$\operatorname{tion}^{22}$. Signe fétiche d'un dilemme anthropologique entre choix et contrainte, le nœud apparaît comme l'objet de l'intersection entre le dessein masqué d'un destin déjà écrit et la fin lente d'une intrigue en apesanteur, mais au devenir implacable.

une seule goutte avant d'avoir effectué avec solennité plusieurs saluts et courbettes, échange de courtoisie que les Africains épris de parade accueillirent en claquant les mains" (ibid. : 113).

20. Ibid. : 107-108.

21. Ibid.

22. Moby Dick (1851) peut être considéré comme la charte mythique de cette vision et Bartleby (1853) comme sa version minimaliste. Massimo Bacigalupo, traducteur italien et commentateur de l'œuvre de Melville, l'a bien fait remarquer: durant l'attaque décisive, la tête de la baleine blanche est définie par un terme assez inusité, "predestinating" et non prédestinée. Cf. Massimo Bacigalupo, «Introduzione », in Herman Melville, Opere, Milano, Mondadori, 1991 : XXXI sq.

\section{Gaetano Ciarcia}




\section{Un temps raréfié et l'opacité du visible}

À bord du San Dominick, toute temporalité est comme suspendue:

«Une fois rapprochés les uns des autres, ces points ne laissaient pas d'apparaître quelque peu contradictoires. Et après? pensa le capitaine Delano en jetant un coup d'œil sur son canot qui se rapprochait - et après ? " ${ }^{23}$

En effet, c'est cet "après" en sursis qui permet un salut provisoire à Delano. Sa montée à bord du navire espagnol s'apparente alors plutôt à la chute dans un monde exotique où les choses cachées n'ouvrent pas la porte de l'Éden, mais deviennent le paravent de l'Enfer qui est celui d'un bateau où s'agite une étrange et diabolique population ${ }^{24}$. Sans le Rover, la chaloupe (cet "après " à l'œuvre), le navire américain ne serait qu'un mirage. Quand parfois, à cause des courants, un promontoire de l'île fait disparaître le Bachelor's Delight de son champ de vision, cette disparition accroît l'inquiétude de son capitaine. La navette entre le Bachelor's Delight et le San Dominick scande le temps pour les pièces blanches et pour celles, noires, de l'échiquier que Melville invente au large du littoral chilien ${ }^{25}$. Cette temporalité flottante aura une fin explosive où la durée jadis immobile éclate et révèle les rouages de sa "mystérieuse " unité : Benito Cereno se lance dans le canot, poursuivi par Babo qui se révèle être l'instigateur machiavélique d'un plan consistant à s'emparer, la nuit tombée, du Bachelor's Delight:

23. Benito Cereno, op. cit. : 111 sq.

24. Sur un autre registre, Cesare Pavese remarquait: «J'irai jusqu’à dire que les marines, les intérieurs, l'angoisse qui étreint le cœur, les voix - tout ce qui compose le décor de la singulière journée vécue par le capitaine Delano sur le San Dominick - sont techniquement analogues au décor de certains épisodes du Purgatoire de Dante - la montée, la veillée, les crépuscules printaniers et les visions -, symboles, au-delà de l'image, d'une conception opposée des choses : la possible spiritualisation angélique" (Cesare Pavese, "Symboles et mythes dans Benito Cereno", Europe, 1991, 69: 111 sq. [Ëd. orig., "Simboli e miti in Benito Cereno", in Herman Melville, Benito Cereno, Torino, Einaudi, 1940.])

25. Dans la réalité géographique l'île de Santa-Maria ne se situe pas loin de la côte mais près de la ville de Concepción. Amasa Delano, navigateur et marchand américain (1763-1823) est l'auteur de Narrative of Voyages and Travels in the Northern and Southern Hemispheres..., Boston, 1817. Delano relate, entre autres, la révolte des esclaves ayant eu lieu à bord du navire espagnol Tryal (le San Dominick de la nouvelle) en 1805, et inclut les actes juridiques relatifs aux faits. Le nom véridique de son bateau était Perseverance. Pour ce qui concerne la réalité historique de la figure de Benito Cereno, dans le texte de Delano, elle apparait sous le nom de Bonito Sereno et de Benito Cereno dans les documents reportés par Delano lui-même; son personnage ainsi que la chute de l'histoire ont été presque complètement inventés par Melville. Dans la version «originale », Cereno est peint comme un être haineux et ingrat ayant tenté de poignarder un prisonnier noir et n'ayant jamais voulu, par la suite, payer à Delano et à ses hommes la récompense promise avant la « reconquête » du Tryal. Il est intéressant de souligner aussi que, d'après la source officielle, Cereno lui-même, qui était déjà leur otage, aurait proposé aux mutins, pour les tranquilliser, de mettre en œuvre leur stratagème. 
"Tout cela, comme ce qui avait précédé et ce qui suivit, se déroula avec une telle rapidité que passé, présent et futur semblaient ne faire qu'un. ${ }^{26}$

Entre sept heures trente du matin et six heures du soir, une partie s'est jouée entre les Noirs et les Blancs. Quand le câble du San Dominick fut tranché,

"... le coup de fouet qui s'ensuivit entraîna le linceul de toile enveloppant l'étrave et révéla soudain, tandis que la coque blanchie se tournait vers le large, la Mort en guise de figure de proue, sous la forme d'un squelette humain, commentaire de craie aux mots de craie tracés en contrebas : Suivez votre chef ${ }^{27}$.

Le squelette est celui de l'armateur Alexandro Aranda, ami de Benito Cereno, propriétaire d'une grande partie des esclaves, et qui fut tué au cours de la mutinerie ayant éclaté à bord du San Dominick. L'accrochage de ses restes à la proue du navire espagnol devait servir d'avertissement aux Espagnols tentés de s'opposer au pouvoir instauré par Babo, après la mutinerie. Durant le procès tenu à Lima quelques semaines après les faits, Benito Cereno relate comment

"... le nègre Babo emmena successivement chacun des Espagnols à l'avant, lui demandant de qui était le squelette, et si, vu sa blancheur, il ne pensait point que ce fût celui d'un Blanc; que chacun des Espagnols se couvrit le visage... $»^{28}$.

Déchiré à jamais par cette incantation, comme hypnotisé, Benito Cereno devient l'esclave de la transe qui lui a été imposée, des actes terribles qui l'ont précédée, de la menace qui l'a suivie.

"Il déclara qu'il était âgé de vingt-neuf années, et brisé de corps et d'esprit; qu'une fois définitivement renvoyé par la Cour, il ne regagnerait point son pays, le Chili, mais qu'il rejoindrait le monastère du mont Agonia; là-dessus signa, ainsi que Son Excellence, fit le signe de croix puis partit comme il était arrivé, dans sa litière, en compagnie du moine Infelez, vers l'Hôpital de Sacerdotes. ${ }^{29}$

Benito Cereno, après avoir vécu l'aliénation, est un homme anéanti.

"L'habit si recherché et coûteux qu'il portait le jour où se produisirent les événements relatés n'avait point été endossé par lui de son plein gré. Autre exemple : cette épée à monture d'argent, symbole apparent du pouvoir despotique. Ce n'était point en fait une épée, mais une épée-fantôme. Le fourreau, artificiellement raidi, était vide. ${ }^{30}$

L'Espagnol a été mis en spectacle par Babo, les symboles de son pouvoir ont été vidés de leur efficacité31. Le noble descendant d'hidalgos conquérant un

26. Benito Cereno, op. cit. : 141.

27. Ibid. : 142.

28. Ibid. : 154. Le squelette d'Aranda, objet d'une hypnose collective, exposé par le «tyran ", rappelle le doublon d'or, cloué au grand mât du Pequod, promis par Achab comme récompense au premier marin qui signalera la présence de Moby Dick.

29. Ibid. : 164 .

30. Ibid. : 167. 
Nouveau Monde, pourtant déjà ancien, est devenu une marionnette entre les mains des esclaves révoltés, expérience définitive de perte de soi à laquelle l'optimisme moderne et la vigueur américaine de Delano ne pourront jamais l'arracher :

«Vous généralisez, Don Benito, et de manière assez lugubre. Mais le passé est le passé; pourquoi moraliser à son encontre? Oubliez-le. Voyez, ce brillant soleil a tout oublié, et cette mer bleue, et ce ciel bleu; ils ont tourné de nouvelles pages.

- Parce qu'ils n'ont point de mémoire, répondit-il, abattu; parce qu'ils ne sont point humains.

- Mais ces doux alizés qui éventent maintenant votre joue, ne viennent-ils point vers vous tel un humain qui panserait vos blessures? Amis chaleureux, amis fidèles, ces alizés.

- Avec leur fidélité, ils ne font que me pousser vers la tombe, Señor, fut la réponse prophétique.

- Vous êtes sauvé, s'écria le capitaine Delano, de plus en plus surpris et peiné; vous êtes sauvé; qu'est-ce qui a jeté une telle ombre sur vous?

- Le nègre. $\aleph^{32}$

Pour Delano, les masques endossés par ses "hôtes" sont les pièces d'un complot qui - celui-ci déjoué - peuvent être rangées dans la "vitrine" d'une mémoire apaisée. Au contraire, pour Benito Cereno avoir été le masque de soi-même a signifié une altération définitive de sa personne ne lui permettant plus de retour en arrière ni de projection dans le futur. Benito Cereno ne pourra pas répondre, sinon comme un simulacre de ce qu'il a été, à l'échange instauré par Delano qui croit l'avoir sauvé. Il est allé au-delà du miroir où il a connu un monde renversé et où le passé est devenu une maladie sans remèdes. L'autre capitaine, échappé sain et sauf de cette expérience, est libre d'inventer sa vérité de vainqueur, mais il demeure incapable d'imaginer et de comprendre la révélation tragique à laquelle l'Espagnol, dépossédé de la clé de sa condition, est désormais enchainé. Benito Cereno est beaucoup moins la vie sauvée par Delano (sauvée d'ailleurs par Benito Cereno lui-même) que la victime de Babo qui,

"Quelques mois plus tard, trâné vers le gibet à la queue d'une mule, [...] connut sa fin silencieuse. Le corps fut réduit en cendres; mais pendant longtemps, la tête, cette ruche de subtilité, fixée sur une perche dans la Plaza, soutint, sans fléchir, le regard des Blancs tandis que les yeux contemplaient par-delà la Plaza l'église Saint-Bartholomé, dans les caveaux de laquelle reposaient alors, comme à présent, les os recouvrés d'Aranda ; et par-delà le pont Rimac, le monastère du mont Agonia, dans lequel trois

31. Un autre exemple de cette spoliation masquée est fourni par la clé, suspendue par une cordelette de soie, qui pend au cou de Benito Cereno. Censée pouvoir ouvrir le cadenas des chaînes d'Atufal, cette clé fait partie du "costume de scène " de l'Espagnol. En effet, c'est le colosse noir, libre de ses mouvements, qui surveille les gestes de son «maître » et de son hôte. En ce sens, le commentaire de Delano, qui, encore ignorant de la vérité, s'exclame à la vue d'Atufal : «Et bien, Don Benito, clef et cadenas: symboles révélateurs en réalité " (ibid.: 87), semble être pourvu par Melville d'un effet sardonique.

32. Benito Cereno, op. cit. : 167. 
mois après avoir été renvoyé par la Cour, Benito Cereno, porté en terre, ne manqua point, cette fois, de suivre son chef. ${ }^{33}$

\section{Un objet d'ethnographie}

À propos de la fin de Babo, un commentateur a souligné: «It is through Babo's lifeless eyes that we meet the "gaze of whites" and look upon St. Bartholomew's church, our vision is once again blurred, preventing us from finally and conclusively unveiling the mystery of "The Negro". "34 $^{34}$ Linterprétation de Benito Cereno comme un drame du regard a d'ailleurs marqué les nombreuses analyses littéraires d'un texte où les ambiguïtés sémiotiques de la situation sont rehaussées par le foisonnement des registres narratiff ${ }^{35}$. D'après les modalités très diverses connotant respectivement le récit des événements, la description du contexte et la vérité finale et officielle du procès, ce n'est pas seulement la position de Delano qui est ironiquement «innocente» (et donc coupable ou fautive de cécité coloniale), mais c'est l'écriture elle-même, avec son scénario et sa charpente linguistique et syntaxique, qui se révèle ne pas être fidèle à son objet. Darren Hughes remarque qu'au-delà des astuces de l'intrigue, l'opacité de la situation est produite aussi par l'utilisation massive de la part de Melville d'expressions conjecturales ainsi que de descriptions construites à travers des doubles négations ou simplement par la réitération du mot «not $»^{36}$.

À propos de descriptions du genre "Not unlike a cenotaph of skulls", présentes à profusion dans Tä̈pi (1846), le premier roman de Melville, Michel Imbert perçoit que « de cette aventure dans les signes étrangers, Melville rapporte quelque chose comme un trait de style : la double négation - peut-être la traduction écrite de ces objets primitifs non identifiables au premier coup d'œil ${ }^{37}$. Cette friction herméneutique entre

33. Ibid. : 168.

34. Darren Hughes, "That First Comprehensive Glance" : The Cinematic Suspense of Benito Cereno", communication présentée à l'Annual Florida State University Literature and Film Conference, Tallahassee, FL, January 2002.

35. Voir Guy A. Cardwell, "Melville’s Gray Story ", Bucknell Review, 1959, 8 : 154-167; William B. Dillingham, Melville's Short Fiction: 1853-1856, Athens, University of Georgia Press, 1977; Bruce H. Franklin, "Apparent Symbol of Despotic Command: Melville's Benito Cereno ", New England Quarterly, 1961, 34 : 462-477; Allen Guttman, "The Enduring Innocence of Captain Amasa Delano", Boston University Studies in English, 1961, 5: 34-45; Charles G. Hoffman, "The Shorter Fiction of Herman Melville", South Atlantic Quarterly, 1953, 52: 414430 ; Mary Rohrberger, "Point of View in "Benito Cereno" : Machinations and Deceptions", College English, 1965, 27: 541-546; Nancy Roundy, "Present Shadows: Epistemology in Melville's "Benito Cereno" ", Arizona Quarterly, 1978, 34: 344-350; John Douglas Seyle, Melville: The Ironic Diagram, Evanston, IL, Northwestern University Press, 1970.

36. Darren Hughes, op. cit.

37. Michel Imbert, "Taïpi. Notice», in Herman Melville, Euvres I, Paris, Gallimard, ("Bibliothèque de la Pléiade»), 1997 : 1226. 
l'existence d'une altérité (chose ou être) et sa définition montre la réticence melvillienne à procéder à une comparaison entre des identités apparemment figées, dans la réalité emportées par leur devenir. Le désarroi de cet observateur "non professionnel », qui est aussi celui de Delano précipité dans les abîmes de ses idées reçues, "met sur la voie de ce lointain intérieur qui n'était pas encore l'objet de l'anthropologie... " ${ }^{38}$. Comme le rappelle encore très pertinemment Imbert : "Melville brouille les typologies au moment où l'école américaine d'ethnologie [...] commence à élaborer des taxinomies qui ne sont pas exemptes des préjugés raciaux $»^{39}$.

Dans Benito Cereno, une telle construction "au négatif" est complémentaire, d'ailleurs, de l'emprise mythologique du récit. Lélan final de Babo à la poursuite, pour lui fatale, de sa proie, pouvant évoquer celui d'Achab, tout comme la tyrannie exercée par le Nègre à bord du San Dominick, sont le miroir d'une domination plus profonde. Babo et Achab défient la monstruosité de la Loi et, tout en s'y perdant, recherchent une délivrance. Par contraste avec le paroxysme palingénésique de cette quête, le Delano de Melville évolue, à son corps défendant, dans un monde imposant l'énigme d'objets dont la matérialité est comme absorbée par leurs signes. Ce n'est pas seulement une vision éclairante des faits qui manque à Delano, mais leur aspect tangible. L'objet est invisible bien qu'étant là, exhibé, comme garantie de retour à une légalité. Si le capitaine américain "does not ask to be a "perceiving center". He is merely living the story, not representing it $»^{40}$. Tout en restant implicites, incertaines et indéfinies, les scènes sont investies $a b$ origine par l'auteur-démiurge du pouvoir de rendre inadéquate toute perception des événements de la part de leur acteur étranger. Une tentative d'interprétation est néanmoins imputable au personnage du capitaine américain qui est aux prises avec un puzzle l'incluant déjà parmi les pièces de la représentation. Sa méthode, consistant à adhérer malgré tout à une logique coloniale dont la présence de Benito Cereno est la trace de l'échec, se précise plutôt comme la vérification anxieuse et obstinée d'une trame dont il prétend incarner la juste narration. En ce sens, l'affrontement dans le texte entre le regard ironique de Melville, son utilisation fictionnelle des sources documentaires et la participation au suspense dans laquelle Delano est engagé pourrait se représenter aux yeux de lecteurs comme un nœud : le récit où des voix multiples s'enchevêtrent, se confondent et, bien sûr, s'égarent.

Dans Hommage à la Catalogne, George Orwell, se souvenant de sa vie dans les tranchées de la guerre civile espagnole, écrit: "C'était sur le

38. Ibid. : 1225 .

39. Ibid. : 1226. Michel Imbert rappelle que l'American Ethnological Society fut fondée en 1842. 40. Darren Hughes, op. cit. 
moment une sale histoire, mais mon esprit y trouve à présent beaucoup à brouter $»^{41}$. Cette transformation des perceptions et du vécu d'antan en souvenirs et domaine intellectuel relève d'une qualité normale de la construction ou de l'irruption dans le champ mental des fragments du passé. Pour le chercheur, la possession anamnésique des faits et des notions dont il est (et aura été) le témoin critique, parfois l'instigateur, implique le devoir heuristique de resituer et restituer en simultané l'objectivation des perspectives cognitives, a priori temporellement distinctes, constitutives de son travail. Le parcours de Delano, explorateur involontaire de l'« ethnologie comparée imaginaire $~^{42}$ melvillienne, descendu dans l'arène de combat où il incarne nécessairement un des personnages ${ }^{43}$, est comparable (bien entendu, d'une manière tout à fait édulcorée) à une situation concrète que j'ai vécue et que j'ai tenté d'analyser au cours de mes enquêtes en pays dogon ${ }^{44}$.

Dans cette région d'élection des études anthropologiques, la responsabilité créatrice de la part des sciences humaines se fonde sur l'existence d'un faux consensus entre Noirs et Blancs, évoquant celui que le capitaine américain trouve à bord du San Dominick. En pays dogon, un antagonisme, fût-il fait de complicité, entre les spécialistes érudits étrangers et leurs informateurs locaux reflète bien une division sociale du travail ethnologique. L'enquêteur apparaît être en équilibre instable entre les positions de maîtrepenseur et d'élève: son autorité institue, en fait, la fonction ethnographique des connaisseurs locaux légitimant, à leur tour, la validité des recherches. Ainsi, les visiteurs se présentant comme experts ou faiseurs d'un savoir dialoguent avec les gardiens/passeurs d'une tradition moderne. Marquée par une sujétion économique aux instances pourvoyeuses, ou censées l'être, de développement (ou plus modestement de profit monétaire), la normalisation de ce contexte est fondée sur un accord indispensable à la distribution des compétences et des intérêts. Ainsi, un mimétisme acculturé de la part des élites locales impliquées dans un marché est devenu nécessaire à son déroulement "correct $»^{45}$. Il s'agit là d'un échange perfor-

41. George Orwell, Hommage à la Catalogne, Paris, Éditions Ivréa, 1982 : 112. [Éd. orig. Homage to Catalonia, Londres, Eric Blair, 1938.]

42. Marc Richir, Melville. Les assises du monde, Paris, Hachette, 1996: 7 sq. L'auteur utilise cette expression à propos du troisième roman de Melville, Mardi (1849).

43. En 1964, Benito Cereno a fait l'objet d'une adaptation théâtrale par le poète et dramaturge américain Robert Lowell; le scénario a été publié dans la trilogie de Lowell, The Old Glory, New York, Farrar Straus \& Giroux, 1968.

44. Voir Gaetano Ciarcia, De la mémoire ethnographique. L’exotisme du pays dogon, Paris, Éditions de l'EHESS, 2003 ("Cahiers de L’Homme») [cf., dans ce numéro, le compte rendu par Éric Jolly, pp. 260-263, ndlr].

45. Cette relation marque aussi les pratiques touristiques, les procédures de préservation et de valorisation des lieux et, bien sûr, les actions inhérentes à la sphère de la coopération internationale. 


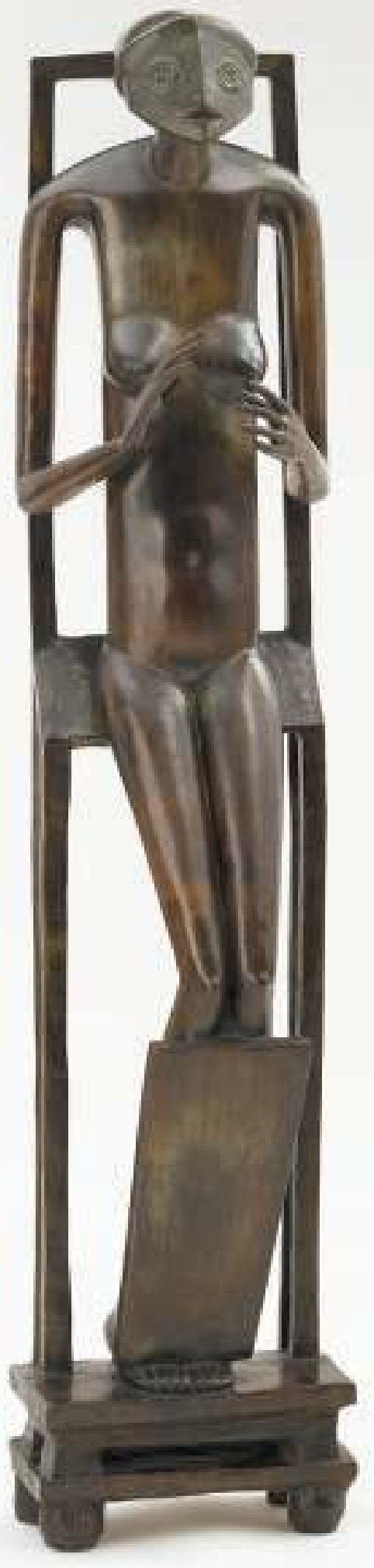


Alberto Giacometti, L'Objet Invisible ou Mains tenant le vide, 1934, bronze (I52,I x 32,7 × 25,4 cm). Legs Louise Reinhardt Smith.

(C) 2003, Digital Image, The Museum of Modern Art, New York/Scala, Florence et ADAGP, Paris 
matif qui dissimule un processus d'apprentissage dirigé par la position historiquement hégémonique des intrus attitrés se voulant être simultanément les initiés savants et les apprentis-héritiers d'un patrimoine.

Dans Benito Cereno, écriture inspirée des crises d'une époque et de ses espaces coloniaux, la dimension théâtrale, mais aussi affective, à l'œuvre dans toute fondation d'un savoir ou d'un ordre qui se voudrait durable, est mise en crise par la révolte des esclaves. Leur mutinerie occultée, qui risque de transformer l'observateur étranger de sauveur potentiel en victime prédestinée, se profile alors comme le négatif d'une efficacité bien concrète, celle nécessaire aux fictions intrinsèques à la domination. Mais, si la représentation inventée de récits et d'images peut métamorphoser leurs créateurs en créatures d'une fiction, "Melville [...] imagine un sujet en mouvement qui part, possédé du désir de la vérité, à la rencontre du non-moi - le monde "objectif", opaque et résistant -, et découvre un mystère absolument irréductible. Et doit affronter l'autorité de l'Autre "46.

Aujourd'hui, au pays dogon comme dans les musées hantés par les déplacements de lieux et de sens d'objets, la reconnaissance de cette "autorité" invite les chercheurs à affronter de manière critique les formes de la réception de leur pensée et de leurs pratiques. En prolongeant, ou en incorporant, cette digression, la figure de Babo, apparemment toujours attentif aux desiderata de ses maitres - mais on pourrait prendre également comme exemple celle, plus récalcitrante, d'Atufal -, peut être lue comme une personnification littéraire de l'«objet nègre». Théâtralement pacifié, jouant l'avatar d'une vocation servile, disponible aux usages de ses possesseurs (en réalité, mascarade jouée à leur intention), il est traversé par un conflit latent (ou en sursis) ayant comme seuls objectifs la vengeance ou le rachat. À l'instar de l'Objet invisible, sculpté par Alberto Giacometti en 1934, son corps "primitif» et poli porte une tête qui est le masque d'un affrontement ${ }^{47}$.

Archive fictionnelle de l'altération occidentale du monde, la transformation romanesque d'un récit autobiographique et d'une source judiciaire scrute les identités des deux capitaines. Ils sont les simulacres vivants du changement de valeurs et de perspectives : le déclin du pouvoir hispanique est relayé par l'insouciance de la puissance américaine. Sans vouloir (et pouvoir) en être l'ethnologue, le capitaine Delano, pendant onze heures,

46. Philippe Jaworski, "Introduction", in Herman Melville, Euvres I, Paris, Gallimard («Bibliothèque de la Pléiade »), 1997 : XII.

47. À propos de cette pièce, dont le titre complet est L'Objet invisible ou Mains tenant le vide, Colin Rhodes a écrit qu' "il s'apparente à certaines sculptures de figures caractéristiques des îles Salomon, en particulier par la pose aux jambes pliées et par le cadre architectural, et pourtant un de ses traits le plus frappant, à savoir le visage aux allures de masque, est dérivé d'un masque militaire de protection fabriqué en France pendant la Première Guerre mondiale ", Colin Rhodes, Le Primitivisme et l'art moderne, Paris, Thames \& Hudson, 1997: 181. [Éd. orig. Primitivism and Modern Art, Londres, Thames \& Hudson, 1994.] 
a vécu une expérience que nous pourrions qualifier d'ethnographique. Il a été confronté au "scandale de la rencontre " 48 avec une humanité - celle de Benito Cereno et de l'équipage du San Dominick - qui, censée être comparable et ouverte à la sienne, s'est révélée porteuse d'une opposition radicale. La présence cruciale, déniée par Delano et dissimulée par les vrais autres, les "barbares", a forgé la seule koinè possible : une langue hachée et obscure pour une comédie masquant la tragédie en train de se jouer. Dans Benito Cereno, immanentes à l'échange entre les deux bateaux/communautés imaginées par Melville, l'ambivalence et l'ambiguïté fondent la relation avec l'Autre et les rapports entre les identités en jeu ${ }^{49}$. Ce n'est pas un hasard littéraire, par exemple, si l'avancée de Delano, se frayant un chemin parmi les Noirs, peut être décrite simultanément comme si elle se déroulait "sous l'escorte d'une garde d'honneur cafre ${ }^{50}$, et comme la progression presque aveugle sur un échiquier d'une pièce blanche égarée au sein des pions adverses.

Le San Dominick, où les mésaventures de la navigation auraient pu instituer une entente originale entre les Blancs et les Noirs, représente la pantomime grotesque et cruelle d'un pouvoir masqué dont le contrôle physique et symbolique a été arraché à ceux qui étaient ses «légitimes " détenteurs; Benito Cereno désormais le sait et Amasa Delano en a l'intuition, mais qui n'arrive pas à s'affirmer comme conscience lucide. Les deux commandants sont aux antipodes de toute fuite exotique vis-à-vis de leur société, le premier a été expulsé de sa condition de maître par "ses " esclaves et l'autre a été propulsé, malgré lui, dans un jeu où la seule possibilité de survie est de ne pas (vouloir) comprendre jusqu'au bout (mais aussi jusqu’à leur but) les règles. Dans ce monde régi par la parodie comme seule forme de salut, nous retrouvons l'exploration melvillienne d'une cosmogonie de l'errance qui distille «le doute [...], l'horreur de l'identité séparée " ${ }^{51}$. Ainsi, à mille lieues de toute expérience (auto)critique du voyage comme rupture sociale, nous sommes confrontés à celle que Daniel Defert, à propos de l'écrivain anglais Robert James Fletcher ${ }^{52}$, a définie comme une "colonisation du soi ». La crise de la temporalité

48. Sur la notion de "scandalo dellincontro etnografico», voir Ernesto de Martino, Furore simbolo valore, Milan, Feltrinelli, 1980.

49. Ce thème se décline dans l'œuvre de Melville, de Taïpi (1846), en passant par Pierre ou les ambiguïtés (1852), à Billy Budd (1891).

50. Benito Cereno, op. cit. : 99.

51. Massimo Bacigalupo, op. cit. : XXVI sq. Ma traduction.

52. Voir Robert James Fletcher, Îles-paradis, îles dillusion. Lettres des Mers du Sud, Paris, Le Sycomore, 1979 [Éd. orig., Isles of Illusion. Letters from the South Seas, Londres, Constable, 1923 ; $1^{\text {re }}$ éd. franç., Lettres des Îles-Paradis, 1928]. Il s’agit d'un recueil de lettres envoyées, entre 1910 et la première moitié des années 1920, par l'auteur, un aventurier anglais qui s'était "perdu " dans les mers du Sud, à un ami londonien. 
reflète ainsi une raréfaction de l'exotisme et nous parle du «brouillage [des identités] jumelé insidieusement dans les multiples canaux du processus colonial ${ }^{53}$. Altération au sein du soi conquérant du Blanc, cette crise de la temporalité masquerait le devenir de sa propre altérité. Benito Cereno, victime soumise, est la figure d'une présence annihilée, alors que celle de Delano exprime les dénégations nécessaires à toute construction de l'objet, à la fabrication ordinaire du savoir-faire de la domination. Mais les "refus» forcés de l'Espagnol à l'égard de son hôte - où résonne toujours la double négation melvillienne, en quelque sorte l'écho du "I would prefer not to" que le clerc Bartleby répond à son employeur - viennent souligner la faillite du séjour de Delano sur le San Dominick, qui ne débouche sur aucun dévoilement d'une vérité. Delano agit dans l'espoir du retour, mais ses détours par les limites scandaleuses de ce qu'il n'arrive pas à saisir ou à accepter signifient tout à la fois la proximité violente de l'Autre et son déni. C'est la situation, avec son dynamisme truqué (cet «après » stagnant et inquiet à la fois), et non le projet de sauvetage entrepris par l'Américain, qui donnera un sens final à ce que le satyre noir masqué annonçait depuis le début du récit. Ce n'est donc pas une formule, à la Bartleby ${ }^{54}$, exprimant la pétrification d'un refus, qui condense le signifié d'une confrontation entre entités prétendument absolues, mais le devenir quotidien - rappelant quelque part la routine ethnographique - de leur rencontre qui, en les altérant, produit un scandale apparemment immobile. Or, la brutalité enfouie dans les formes diverses d'observation des mœurs qu'on scrute sans toujours pouvoir les comprendre («je voyais tout et je ne comprenais rien", dit Tommo, le narrateur du premier roman de Melville, Tä̈pi, à propos de sa mystérieuse captivité chez les indigènes) imprègne aussi la relation entre l'ethnologue et ses "visités». Le regard du chercheur en quête de l'objet de son voyage - celui de Malinowski débarquant sur la grève de "son" île, par exemple - serait alors aussi bien dirigé par une observation se voulant participante vis-à-vis des «impondérables de la vie quotidienne» des Autres que par une distance "participée». En ce sens, le choix de s'associer (ou de se joindre) à la culture d'autrui intègre, en la dissimulant partiellement, une présence éloignée, vécue plus comme un positionnement stratégique et une contrainte existentielle que comme une prémisse méthodologique librement appliquée. Dans une optique éminemment anthropologique, nous serions confrontés à la nécessité phy-

53. Daniel Defert, "Un cas d'hébridisation : Robert James Fletcher", Journal de la Société des Océanistes, 1981, 70-71:130.

54. Cf. Gilles Deleuze, "Bartleby, ou la formule ", in Herman Melville, Bartleby. Les Îles enchantées, Paris, Flammarion, 1989: 171-203. 
sique, avant d'être épistémique, de ce "dédoublement de la moralité "55 que Victor Segalen, dans son enquête sur la vie de Rimbaud à Aden, prenait en compte pour tenter d'expliquer le silence du poète dans les lieux trop réels de ses voyages ${ }^{56}$.

L'ethnologie étant une tradition discursive dont les Autres sont l'objet intellectuel et muséographique, il est intéressant de noter que l'idée de participation du chercheur aux représentations et aux conceptions des Autres n'est entrée que rarement en concurrence avec le désaveu marqué au sein de la discipline de la notion lévybruhlienne de participation, mode de pensée archaïque attribué aux peuples " primitifs ». Certes, la fondation scientifique de l'observation, avec son devoir corollaire d'authenticité morale, a évité tout danger d'une contagion sémantique entre les deux types de participation: celle "ethnographique» aux mœurs d'autrui, et celle "prélogique » qui aurait été affublée de façon erronée à une altérité fictive. Mais alors la «lente et progressive dépersonnalisation intime que suscitent la raréfaction de la communication verbale [...] la solitude, la langueur, la moiteur, la maladie des Tropiques, l'alcool, l'environnement colonial, en un mot: la colonisation du soi ${ }^{57}$ peuvent dire quelque chose aux anthropologues. La vérité ficta et mobile des mondes sociaux s'exprime aussi à travers la communication aveugle séparant Delano et ses Autres, qui lui sont si physiquement proches. Sa participation observée (ou plutôt surveillée) par ses hôtes aux symptômes qu'il vit et qu'il voudrait détecter à bord du San Dominick révèle la réalité de son être agi dans un monde qu'il vaut mieux, pour lui, regarder en étranger pour que l'«autorité » de ses objets/sujets ne devienne pas outre mesure lisible et donc ouvertement hostile.

C'est la diversité des coutumes qui sollicite, bien sûr, le regard. Mais cette altérité que la logique comparative devrait rendre moins absolue après l'avoir édifiée comme exotique est tout autant une donnée préexistant à la rencontre qu'une découverte partagée s'inventant à partir du moment clé, mais rare, masqué ou en sursis, où toute aspiration à une compréhension intégrale s'avère un leurre ou un danger. L'œil de l'observateur, à l'instar de sa "mauvaise " lecture des signes, semble participer alors à la création d'une distance qui n'est plus seulement celle préalable à l'échange, mais aussi celle nécessaire, dans l'immédiat, à son déroulement, à son objectivation.

55. Victor Segalen, Le Double Rimbaud, in CEuvres I, Paris, Robert Laffont, 1995 : 498. [Éd. orig. Mercure de France, 15 avril, 1906].

56. Voir Jean Jamin, "Note sur l'étrange cas de Robert James Fletcher ", Journal de la Société des Océanistes, 1981, 70-71:131-132, où l'auteur fait allusion à la « disparition » de l'écrivain Robert James Fletcher au retour de ses voyages, contrepoint existentiel (et géographique) au silence littéraire rimbaldien.

57. Daniel Defert, op. cit. : 129. 
MOTS CLÉS/KEYWORDS : esclavage/slavery - Herman Melville - masque/mask - observation participante/participant observation - situation ethnographiquelethnographic situation - fiction.

RÉSUMÉ/ABSTRACT

Gaetano Ciarcia, L'objet invisible, ou le gambit du capitaine Delano. - Les objets de la recherche ethnomuséographique sont ici scrutés à travers la lecture critique du conte Benito Cereno. Narration romanesque d'un événement qui s'est réellement produit durant l'époque de la traite négrière, ce texte d'Herman Melville met en scène l'altération des identités à l'œuvre dans le processus colonial. Par son recours à l'utilisation fictionnelle des sources documentaires, le récit, brouillant la reconstruction historique et l'invention littéraire, interroge aussi les dénégations nécessaires aux savoir-faire de la domination et de ses masques.
Gaetano Ciarcia, The Invisible Object, or the Captain's Gambit. - The subjects of research in the ethnological museum sciences are examined through a critical reading of Benito Cereno. Herman Melville's narration of an event that actually happened during the slave trade hinges on the altering of identities via the colonial process. By using documents for the purpose of fiction, this story blurs the bounds between historical reconstruction and literary fabrication. It inquires into the disclaimers that are necessary to know how to exercise domination and wear the master's masks. 